\title{
Perfil tomográfico de pacientes atendidos com acidente vascular encefálico em hospital de urgência em Teresina - PI
}

\author{
Tomographic profile of patients attended with brain vascular accident in an emergency hospital in \\ Teresina - PI
}

Perfil tomográfico de paciente atendidos con accidente vascular cerebral en un hospital de

urgencias de Teresina - PI

Recebido: 06/12/2021 | Revisado: 10/12/2021 | Aceito: 12/12/2021 | Publicado: 21/12/2021

\author{
Yúllia Alves Santos Rufino \\ ORCID: https://orcid.org/0000-0003-4736-6459 \\ Centro Universitário Uninovafapi, Brasil \\ E-mail: yullia_santos@hotmail.com \\ Ianna Veloso \\ ORCID: https://orcid.org/0000-0002-3089-8347 \\ Centro Universitário Uninovafapi, Brasil \\ E-mail: iannavelosog@gmail.com \\ Williams Cardec da Silva \\ ORCID: https://orcid.org/0000-0002-7167-4876 \\ Centro Universitário Uninovafapi, Brasil \\ E-mail: willcardec@hotmail.com
}

\begin{abstract}
Resumo
Objetivou-se com este estudo conhecer padrão tomográfico e epidemiológico mais prevalentes dos pacientes admitidos com acidente vascular encefálico (AVE) no Hospital de Urgência de Teresina - HUT no ano de 2020. Trata-se de um estudo epidemiológico, observacional quantitativo, transversal, retrospectivo, realizado por coleta de dados em prontuários eletrônicos. Os resultados revelaram que, dos 384 casos, 73,6\% tiveram AVE isquêmico (AVEi), a região cerebral mais acometida no AVEi foi a região fronto-parietal com 36,4\% dos casos e no AVEh a região parieto- temporal com $28,7 \%$. Os principais fatores de risco foram Hipertensão Arterial (62,5\%) e Diabete Melito $(31,2 \%)$. O intervalo de tempo entre a admissão do paciente e a realização da Tomografia Computadorizada (TC) variou entre 30min a 3h. Das intervenções apenas 3\% usaram trombolítico. A partir dos resultados conclui-se que o perfil da população estudada não difere daquele, usualmente, encontrado na literatura e que houve dificuldade em determinar o tempo de início dos sintomas e, com isso, a indicação da terapia trombolítica.
\end{abstract}

Palavras-chave: Acidente vascular encefálico; Manejo médico; Tomografia computadorizada.

\begin{abstract}
The objective of this study was to know the most prevalent tomographic and epidemiological pattern of patients admitted with a stroke at the Emergency Hospital of Teresina - HUT in 2020. This is an epidemiological, quantitative observational, cross-sectional study. retrospective, performed by collecting data from electronic medical records. The results revealed that, of the 384 cases, $73.6 \%$ had ischemic CVA (iCVA), the brain region most affected in CVAi was the fronto-parietal region with $36.4 \%$ of the cases and in CVA the parietotemporal region with $28.7 \%$. The main risk factors were Hypertension (62.5\%) and Diabetes Mellitus (31.2\%). The time interval between patient admission and the performance of Computed Tomography (CT) ranged from 30min to $3 \mathrm{~h}$. Of the interventions, only $3 \%$ used thrombolytics. From the results, it can be concluded that the profile of the studied population does not differ from that usually found in the literature and that there was difficulty in determining the time of onset of symptoms and, therefore, the indication for thrombolytic therapy.
\end{abstract}

Keywords: Stroke; Medical management; Computed tomography.

\section{Resumen}

El objetivo de este estudio fue conocer el patrón tomográfico y epidemiológico más prevalente de los pacientes ingresados por ictus en el Hospital de Urgencias de Teresina - HUT en el año 2020. Se trata de un estudio epidemiológico, observacional, cuantitativo, transversal, retrospectivo, realizado mediante colecta datos de registros médicos electrónicos. Los resultados revelaron que, de los 384 casos, el 73,6\% tenía CVA isquémico (iCVA), la región cerebral más afectada en CVAi fue la región frontoparietal con el 36,4\% de los casos y en el CVA la región parietotemporal con el 28,7\%. Los principales factores de riesgo fueron Hipertensión (62,5\%) y Diabetes Mellitus $(31,2 \%)$. El intervalo de tiempo entre la admisión del paciente y la realización de la tomografía computarizada (TC) 
osciló entre 30min y 3h. De las intervenciones, solo el 3\% utilizó trombolíticos. De los resultados se puede concluir que el perfil de la población estudiada no difiere del que habitualmente se encuentra en la literatura y que existió dificultad para determinar el momento de aparición de los síntomas y, por tanto, la indicación de la terapia trombolítica.

Palabras clave: Ictus; Administración medica; Tomografía computarizada.

\section{Introdução}

O Acidente Vascular Encefálico (AVE) se caracteriza por déficit neurológico súbito, de origem vascular podendo durar até mais de 24 horas ou resultar em morte. É dividido em dois tipos: Isquêmico e Hemorrágico, destacando-se o isquêmico como o mais frequente na população brasileira, podendo chegar a até $85 \%$ dos casos (Rolim et al, 2017). O AVE é considerado uma emergência clínica e um atraso em seu diagnóstico e tratamento pode ter complicações clínicas importantes, portanto, é prioritário que os profissionais emergencistas estejam familiarizados com as atualizações no manejo desses pacientes (Figueiredo et al, 2012)

De acordo com os dados do Sistema de Informação Hospitalar (SIH) do Ministério da Saúde (MS) o AVE é considerado um dos principais motivos de internação no Sistema Único de Saúde (SUS) sendo uma doença de grande impacto social, acometendo principalmente adultos e idosos. Por causar mortes e incapacidades, constitui-se a primeira causa de mortalidade no Brasil e a segunda no mundo, competindo com as doenças isquêmicas do coração (Brasil, 2020).

Em um paciente com quadro suspeito de AVE a avaliação clínica é incapaz de distinguir entre lesão isquêmica ou hemorrágica. Diante disso, os estudos de imagem são usados para excluir hemorragia no paciente com AVC agudo, para avaliar o grau de lesão cerebral e para identificar a lesão vascular responsável pelo déficit isquêmico (Lima et al, 2012). Algumas tecnologias avançadas de tomografia computadorizada (TC) e ressonância magnética (MRI) são capazes de distinguir entre o tecido cerebral que está irreversivelmente infartado e aquele que é potencialmente recuperável, permitindo assim distinguir o manejo terapêutico para cada situação (Botelho et al., 2016).

A TC é o principal método diagnóstico de imagem a ser solicitado, pois além de definir se a lesão é isquêmica ou hemorrágica, permite verificar a extensão e a severidade do acidente. A interpretação desse exame por uma equipe especializada permite a distinção entre as hipóteses diagnósticas possíveis, auxilia a decisão sobre a indicação clínica da terapêutica trombolítica, além de nortear as demais condutas clínicas e a utilização de outras tecnologias adequadas a cada caso (Mourão et al, 2017).

Os estudos de imagem cerebral permitem a detecção de lesões hemorrágicas, a avaliação do grau de lesão cerebral e elucidação dos mecanismos de isquemia ou hemorragia intracraniana. Permitem uma melhor seleção de pacientes que podem ser tratados com terapia de reperfusão, através da identificação de regiões do cérebro com tecido viável, risco de transformação hemorrágica e oclusões das grandes artérias que podem ou não ser passíveis de terapia. (Caplan, 2020)

A terapia de reperfusão consiste no reestabelecimento do fluxo sanguíneo do tecido cerebral, e é a conduta preconizada no AVE isquêmico, devendo ser instituída precocemente, uma vez que seu benefício diminui continuamente ao longo do tempo. Por esse motivo é de suma importância determinar se o paciente é elegível ainda nas primeiras horas da avaliação. (Filho et al. 2020)

A TC é o estudo de imagem de primeira escolha na maioria dos centros devido à ampla disponibilidade, tempos de varredura rápidos e facilidade de detecção de hemorragia intracraniana, é a modalidade de imagem mais comum usada para triagem de pacientes com AVC agudo em todo o mundo. Esse método de imagem é indispensável quando existem contraindicações absolutas à RM, ressaltando-se que a TC sem contraste é o método mais amplamente utilizado por ter custo mais baixo, maior disponibilidade e menor tempo necessário para realização (Magalhães et al, 2017).

O AVE, seja de natureza hemorrágica ou isquêmica, tem a capacidade de levar a resultados devastadores e 
debilitantes para o paciente, o que não só tem implicações diretas do ponto de vista da saúde, mas seus efeitos são duradouros e impactam a comunidade como um todo. Por essa razão, dada a importância do diagnóstico precoce e da terapêutica adequada, este estudo visa observar o padrão tomográfico mais prevalente bem como analisar fornecer dados que permitam a correlação clínica e a avaliação da eficácia terapêutica instituída e sua adequação às recomendações de manejo do AVE na literatura vigente.

\section{Metodologia}

Realizou-se um estudo epidemiológico observacional quantitativo, transversal, retrospectivo, no qual os pesquisadores não tiveram controle ou interferência alguma sobre a obtenção dos resultados, as informações foram coletadas em um único momento da pesquisa, e os dados foram obtidos através de relatos e registros de fatos já ocorridos (Pereira et al, 2018). Foram analisados 389 prontuários eletrônicos de pacientes com diagnóstico clínico de AVC admitidos no Hospital de Urgência de Teresina - HUT no período de 1 de janeiro a 31 de dezembro de 2020.

As informações dos pacientes catalogados na Classificação Internacional de Doenças (CID-10) de Acidente vascular cerebral não especificado (I64) foi fornecida pelo setor de Tecnologia de Informação do HUT. Dados sócio-demográficos e clínicos foram extraídos dos prontuários tendo como finalidade descrever o perfil dos pacientes, sendo analisadas as seguintes informações: idade, sexo, história clínica de comorbidades e/ou tratamentos prévios, exames de imagem, tempo de internação, principais sintomas. Os dados foram armazenados e analisados através da planilha EXCEL e do pacote estatístico SPSS® 19.0 (statisticial package for the social). Os resultados foram compilados em tabelas e gráficos para quantificação das prevalências.

O estudo foi realizado conforme a Resolução 466/2012. O projeto foi avaliado e aprovado pelo Comitê de Ética em Pesquisa do Centro Universitário UNINOVAFAPI, Projeto: CAAE - 45518921.8.0000.5210 de 21 de maio de 2021.

\section{Resultados}

Dos pacientes analisados na pesquisa, 73,6\% $(n=283)$ apresentaram AVE isquêmico (AVEi) e 26,4\% (n=101) AVE hemorrágico (AVEh) (gráfico I). Em relação a região cerebral mais acometida, no AVEi prevaleceu a região fronto-parietal com 36,4\% ( $\mathrm{n}=103)$ dos casos e no AVEh a região parieto- temporal foi a mais acometida, representando $28,7 \%$ ( $\mathrm{n}=29)$ casos (Gráfico 1).

Gráfico 1 - Distribuição de pacientes com AVE segundo o tipo.

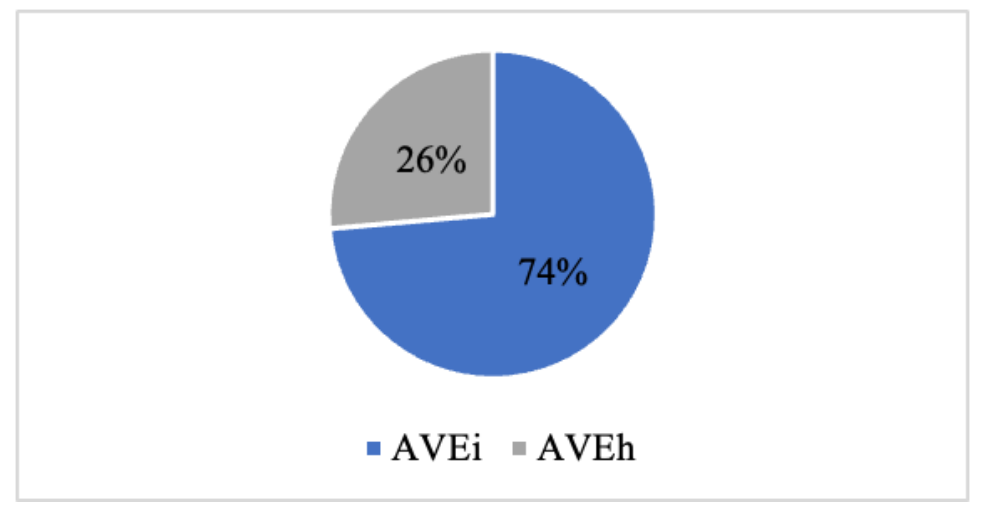

Fonte: Dados da pesquisa (2021). 
Gráfico 2 - Distribuição de pacientes acometidos com AVE segundo a localização da lesão no cérebro.

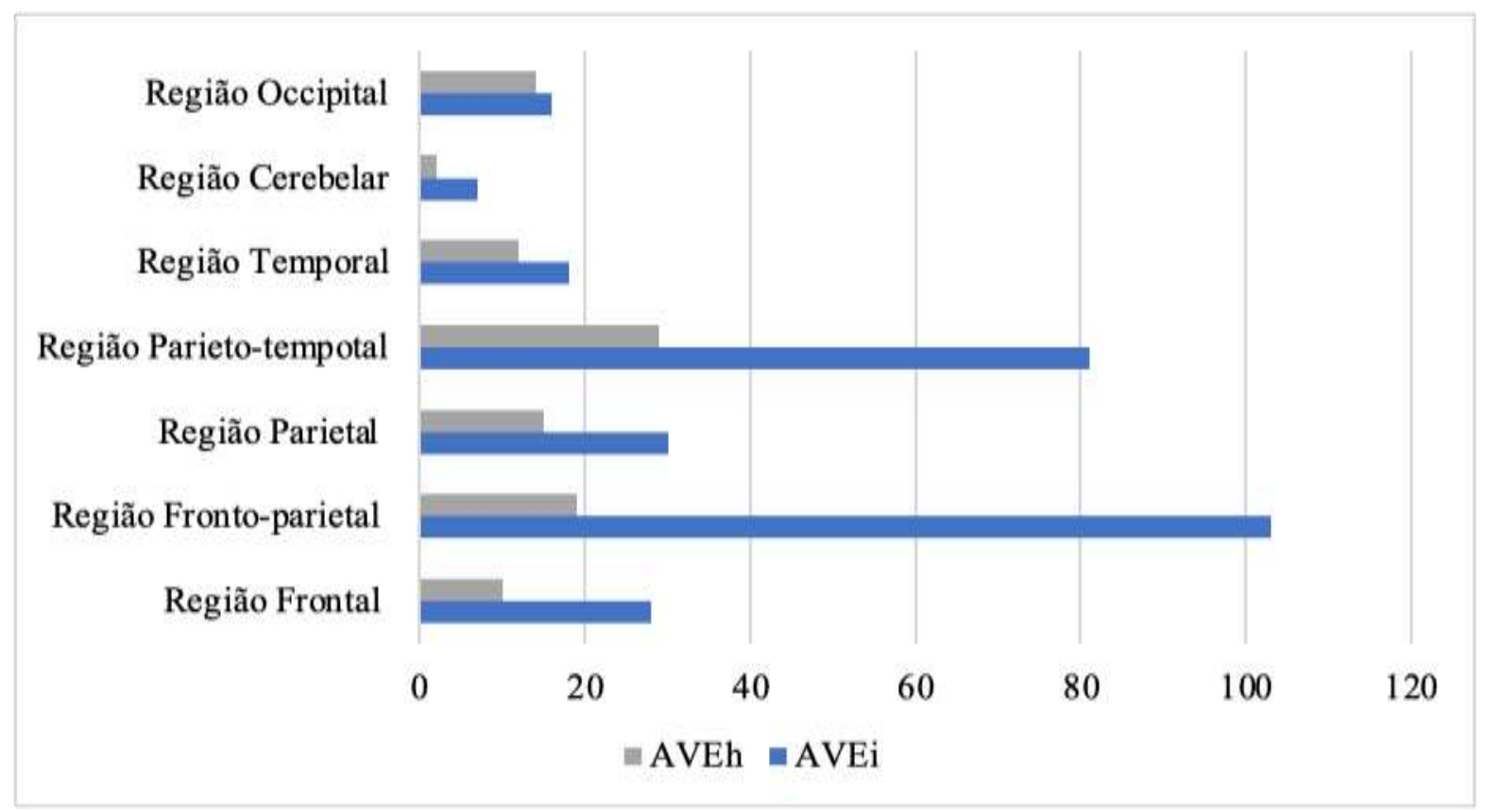

Fonte: Dados da pesquisa (2021).

Como mostra a Tabela 1, a média de idade foi na faixa dos 60 a 69 anos, correspondendo à 26,8\% (n=103) dos casos com amplitude de 20 a 95 anos. De modo geral houve uma equivalência entre os sexos, sendo 48,6\% (n=187) mulheres e $51,3 \%(\mathrm{n}=197)$ homens, não encontrando diferença significativa da média de idade entre os sexos.

Tabela 1 - Distribuição de pacientes acometidos com AVE segundo idade e sexo.

\begin{tabular}{lccc}
\hline VARIAVÉIS & $\begin{array}{c}\text { AVEi } \\
(\mathbf{n = 2 8 3})\end{array}$ & $\begin{array}{c}\text { AVEh } \\
(\mathbf{n = 1 0 1})\end{array}$ & \% \\
\hline IDADE (anos) & & & 6,5 \\
$20-29$ & 15 & 10 & 8,3 \\
$30-39$ & 19 & 13 & 11,9 \\
$40-49$ & 27 & 19 & 12,5 \\
$50-59$ & 34 & 14 & 26,8 \\
$60-69$ & 83 & 14 & 20 \\
$70-79$ & 63 & 11 & 13.8 \\
$>80$ & 42 & 54 & 48,6 \\
Feminino & & 51,3 \\
Masculino & 133 & 47 & 51,3 \\
\hline
\end{tabular}

Fonte: Dados da pesquisa (2021).

Dentre os fatores de risco identificados, a Hipertensão Arterial Sistêmica (HAS) apresentou maior prevalência com 62,5\% ( $\mathrm{n}=240)$ dos casos, seguido da Diabetes (DM) com 31,2\% (n=120) e cardiopatias com 14\% ( $=54)$. Em relação aos sintomas associados $25 \%(n=96)$ dos pacientes apresentaram hemiparesia, $19 \%(n=76)$ cefaleia, $15 \%(n=58)$ disfasia e $12,7 \%$ $(\mathrm{n}=49)$ desvio da rima labial. (Tabela 2). 
Tabela 2 - Distribuição dos pacientes atendidos com AVE segundo comorbidades e sinais e sintomas.

\begin{tabular}{cccc}
\hline VARIÁVEIS & AVEi & AVEh & \\
\hline COMORBIDADES* & & & $\%$ \\
HAS & 182 & 58 & 62,5 \\
DM & 92 & 28 & 31,2 \\
Cardiopatia & 43 & 11 & 14 \\
Outros & 32 & 8 & 13,5 \\
Não informado & 31 & 21 & 19,8 \\
SINAIS E SINTOMAS* & & & 7,5 \\
Cefaleia & 41 & 35 & 10,9 \\
Vômitos & 15 & 14 & 25 \\
Perda da consciência & 22 & 20 & 15,3 \\
Hemiparesia & 55 & 38 & 15,1 \\
Desvio da rima labial & 41 & 18 & 15 \\
Disfasia & 43 & &
\end{tabular}

*Pode haver mais de uma resposta para um mesmo paciente. Fonte: Dados da pesquisa (2021).

O intervalo de tempo entre a admissão do paciente e a realização da Tomografia Computadorizada (TC) variou entre 30 min a $3 \mathrm{~h}$, sendo que $45 \%(n=173)$ realizaram a TC em até $1 \mathrm{~h}$ após a admissão, 34,6\% (n=133) entre $1 \mathrm{~h}$ e $2 \mathrm{~h}$ e $20 \%(\mathrm{n}=78)$ entre $2 \mathrm{~h}$ e $3 \mathrm{~h}$ (Gráfico 3). O intervalo de tempo entre o início dos sintomas e o primeiro atendimento só foi possível determinar em 148 casos, variando de 1h a 7 horas, na maioria dos prontuários $(n=236)$ não havia informações sobre a hora do início dos sintomas.

Gráfico 3 - Distribuição dos pacientes acometidos com AVE segundo o intervalo de tempo entre a admissão e a realização da Tomografia computadorizada (TC).

\section{Intervalo de tempo entre a admissão e realização}

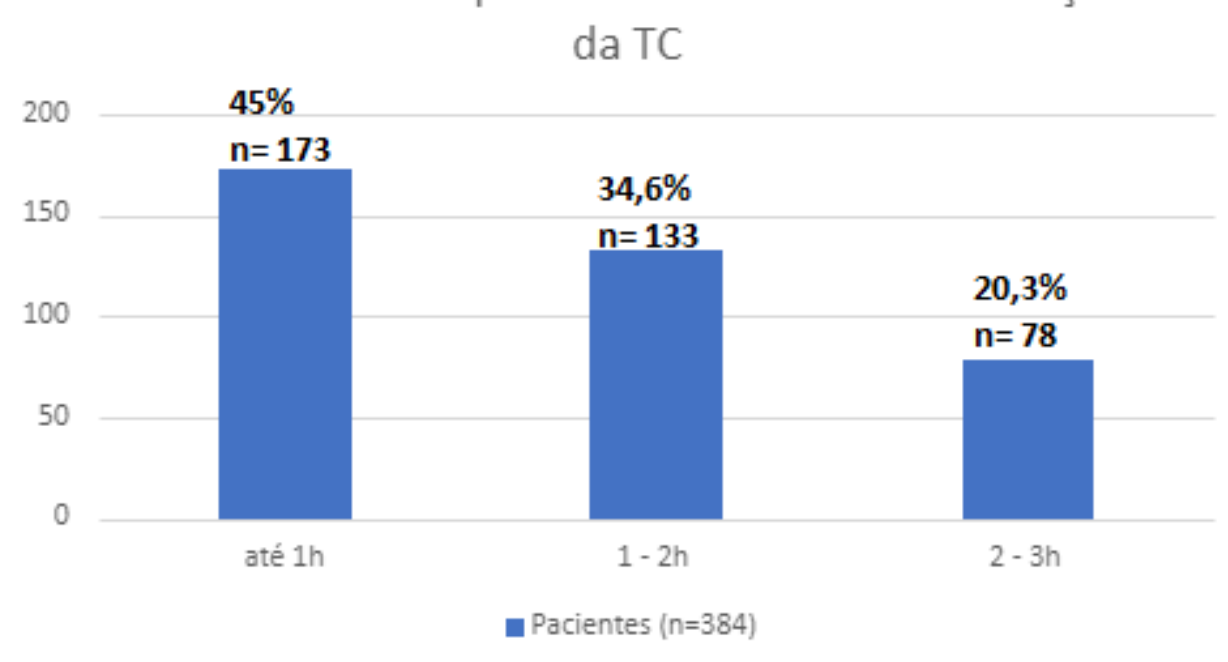

Fonte: Dados da pesquisa (2021).

Em relação ao tempo de permanência no hospital, a quantidades de dias variou entre 1 e $>15$ dias, sendo que a maioria dos pacientes permaneceu internado entre 1 a 5 dias $(36,4 \% ; n=140)$ e apenas $13,8 \%(n=53)$ permaneceu no hospital por mais de 15 dias. Sobre as intervenções realizadas, 55,7\% ( $\mathrm{n}=214)$ necessitaram apenas de suporte clinico, 18\% (n=69) realizaram cirurgia, sendo a maioria $(65,2 \%, n=45)$ observada em pacientes diagnosticados com AVEh. Foram transferidos para outro 
hospital 22,9\% $(\mathrm{n}=88)$ e apenas 3,4\% $(\mathrm{n}=13)$ foram submetidos a terapia de reperfusão. Quanto ao desfecho, 42,1\% (n=162) receberam alta hospitalar e $35,9 \%(n=138)$ foram a óbito. (Tabela 3$)$

Tabela 3 - Distribuição dos pacientes acometidos com AVE segundo permanência no hospital (dias), intervenções e desfecho.

\begin{tabular}{|c|c|c|c|}
\hline VARIÁVEIS & AVEi & AVEh & \\
\hline $\begin{array}{c}\text { PERMANÊNCIA NO HOSPITAL } \\
\text { (DIAS) }\end{array}$ & & & $\%$ \\
\hline 1 a 5 & 116 & 24 & 36,4 \\
\hline 6 a 10 & 97 & 18 & 29,9 \\
\hline 11 a 15 & 55 & 24 & 20,5 \\
\hline$>15$ & 15 & 33 & 12,5 \\
\hline \multicolumn{4}{|l|}{ INTERVENÇÕES } \\
\hline Suporte clínico & 185 & 29 & 55,7 \\
\hline Terapia de reperfusão & 13 & - & 3 \\
\hline Cirurgia & 22 & 51 & 19 \\
\hline \multicolumn{4}{|l|}{ DESFECHO } \\
\hline Alta & 134 & 28 & 42,1 \\
\hline Óbito & 66 & 52 & 30,7 \\
\hline Transferência & 83 & 21 & 27 \\
\hline
\end{tabular}

Fonte: Dados da pesquisa (2021).

\section{Discussão}

O Acidente Vascular Encefálico (AVE) está em segundo lugar entre as principais causas de morte no Brasil, atrás apenas dos óbitos por doenças cardíacas isquêmicas (Brasil, 2021). Com base nos mecanismos fisiopatológicos pode ser definido como isquêmico e hemorrágico, e de acordo com a literatura, a grande maioria dos casos são de etiologia isquêmica (Martins, M, 2010), como foi encontrado nos pacientes do estudo em questão, dos quais 73,6\% apresentaram Acidente Vascular Encefálico Isquêmico (AVEi). Em relação a região cerebral mais acometida, de acordo com os laudos tomográficos analisados no estudo, há predomínio de lesões na região parietal, tanto em AVE isquêmicos como em hemorrágicos, segundo Kulesh et al. (2019), lesões nesta região são mais frequentes uma vez que acidentes vasculares ocorrem com maior frequência no território de irrigação da artéria cerebral média, que vasculariza a maior parte da região superior e lateral dos hemisférios.

O AVE é uma doença cerebrovascular com elevada morbimortalidade dentre as desordens vasculares, acometendo principalmente idosos, no nosso estudo, a prevalência concentrou-se na faixa etária de 60-69 anos, concordando com Cavichioli et al. (2014), o qual refere que o risco de AVE dobra após os 50 anos de idade a cada década, sendo a idade avançada um importante fator de risco. Na amostra analisada no estudo, não houve diferença significativa entre os sexos, na literatura não há um consenso quanto ao sexo mais acometido no AVE, no entanto o risco de as mulheres desenvolverem AVE isquêmico após menopausa aumenta, uma vez que o estrógeno é fator protetor contra aterosclerose (Soeiro, 2018)

A prevalência do AVE está diretamente relacionada com os fatores de risco e a dificuldade de controle dos mesmos, dificultando a prevenção do evento agudo (Bensenor et al, 2015). Nesse contexto, a Hipertensão Arterial Sistêmica (HAS) é a comorbidade mais prevalente nos pacientes acometidos com AVE e estima-se que seu controle e tratamento sejam responsáveis por uma redução de cerca de $20 \%$ da mortalidade cardiovascular (Cavichioli et al, 2014). No hospital estudado, $240(62,5 \%)$ pacientes eram hipertensos, sendo observando maior frequência de HAS nos com AVEi (n=182). Os outros fatores de risco identificados foram Diabetes Mellitus $(31,2 \%)$ e cardiopatia (14\%). 
No estudo em questão não houve diferença significativa dos sinais e sintomas entre AVEi e AVEh, sendo a hemiparesia $(25 \%)$, cefaleia $(19 \%)$ e desvio da rima labial $(15,3 \%)$ os mais frequentes em ambos os tipos. Diante disso, é de suma importância os estudos de imagem, uma vez que são usados principalmente para excluir hemorragia no paciente com AVE agudo, já que a avaliação clínica é incapaz de distinguir entre lesão isquêmica ou hemorrágica (Zerna et al. 2018).

A isquemia cerebral quando persiste por mais de 4 horas provoca lesões neurológicas permanentes, por isso é importância da busca imediata de assistência médica após início dos sintomas, para não exceder as janelas terapêuticas para tratamento trombolítico (Yamashita et al., 2004). Na maioria dos prontuários avaliados nesta pesquisa (n=236) não foi possível precisar o intervalo de tempo entre o início dos sintomas e o primeiro atendimento por não descrição ao prontuário médico, nos demais prontuários $(\mathrm{n}=148)$ o intervalo de tempo entre início dos sintomas e assistência médica variou de $1 \mathrm{~h}$ a $7 \mathrm{~h}$.

No hospital estudado, o intervalo de tempo entre a admissão do paciente e a realização da Tomografia Computadorizada (TC) variou entre 30min a $3 \mathrm{~h}$, sendo que a maioria dos pacientes $(45 \%$; $\mathrm{n}=173)$ realizaram a TC em até $1 \mathrm{~h}$ após a admissão. A importância da TC nas primeiras horas de um AVE isquêmico deve ser reforçada, visto que, as alterações encontradas neste exame poderão determinar ou não a indicação para a terapêutica fibrinolítica. Desta forma, é importante que todos os profissionais envolvidos na abordagem do paciente com AVE estejam familiarizados com os sinais tomográficos precoces de um AVC isquêmico (Abreu, 2010).

Das intervenções avaliadas no estudo, o que mais chamou atenção foi o uso de trombolítico, do total de pacientes com diagnóstico confirmado de AVEi (283) apenas 3\% $(n=13)$ foram trombolisados. De acordo com Fonseca et al. a correta indicação e utilização do Alteplase (rt- PA) diminui a morbidade em 30\%, no entanto seu uso encontra barreiras que vão desde a demora em chegar ao hospital quando do início dos sintomas, ultrapassando o período da janela terapêutica para uso do trombolitico, considerado entre $3 \mathrm{~h}$ e $4,5 \mathrm{~h}$, até a insegurança dos médicos pelo risco de hemorragia intracerebral e incertezas quanto ao benefício do tratamento. No entanto, não fica claro no prontuário analisados os motivos que contraindicavam a terapia, mesmo naqueles que atendiam ao tempo estabelecido na literatura, concluindo-se pelo já exposto, que a grande dificuldade foi reconhecer o tempo do início dos sintomas.

O AVE é uma doença com alta morbimortalidade, sendo que o número de óbito pela doença no Brasil é um dos maiores dentre os países da América Latina. O nosso estudo, em relação ao óbito, mostrou uma taxa de mortalidade de 30,7\% (118), com associação significativa entre o óbito e o tipo de AVEh, 51,4\% contra 23,3\% de óbitos em pacientes com AVCi. De acordo com a literatura pesquisada, o AVEh apresenta quadro clínico mais grave e com maior mortalidade (Zerna et al. 2018).

\section{Conclusão}

Diante do exposto, conclui-se que os aspectos epidemiológicos do estudo estão de acordo com a literatura, o tipo de AVE mais frequente foi o isquêmico, a faixa de idade de maior ocorrência foi de idosos, sem predomínio de sexo. Os fatores de risco principais foram HAS e DM, sendo a relação entre HAS e AVEi estatisticamente relevante. Em relação ao perfil de atendimento, um aspecto negativo foi a dificuldade em determinar o tempo de início dos sintomas e, com isso, a indicação da terapia trombolítica ficou comprometida. Entretanto, uma vez levantada a suspeita de AVE, o tempo até a realização da TC e a confirmação diagnóstica foi eficiente.

Dessa forma é importante enfatizar a realização de atividades educativas nos serviços de saúde e na população em geral, com divulgação de informações sobre os principais sinais e sintomas relacionados ao AVE, ressaltando a importância da procura imediata de um serviço especializado. 
Research, Society and Development, v. 10, n. 17, e49101724387, 2021

(CC BY 4.0) | ISSN 2525-3409 | DOI: http://dx.doi.org/10.33448/rsd-v10i17.24387

\section{Referências}

Abreu, T. T. (2010). Early CT signs in acute ischaemic stroke. Medicina Interna. 9(1), 45-51. https://www.spmi.pt/revista/vol09/sinaisavc.pdf

Bensenor, I. M.; Goulart, A. C., Szwarcwald, C. L., Vieira, M. L. F. P., Malta, D. C. \& Lotofo, P. A.(2015). Prevalência de acidente vascular cerebral e de incapacidade associada no Brasil: Pesquisa Nacional de Saúde - 2013. Arq. Neuro-Psiquiatr. 73(9) 746-750 https://doi.org/10.1590/0004-282X20150115

Botelho, T. S., Neto, C. D. M.; Araújo, F. L. C. \& Assis, S. C. (2016). Epidemiology ofstroke in Brazil. Temas em Saúde. 16(2), 361-377. https://temasemsaude.com/wp-content/uploads/2016/08/16221.pdf

Brasil. (2020). Ministério da Saúde. Secretaria de Atenção à Saúde - SAS; Departamento de Regulação, Avaliação e Controle - DRAC - Coordenação-Geral de Sistemas de Informação - CGSI. Manual do Sistema de Informações Hospitalares do SUS (SIH/SUS). http://dtr2001.saude.gov.br/sas/download/$\% 20$ VERSAO $\% 20$ FINAL-b.pdf

Caplan, L. R. (2020). Overview of the evaluation of stroke. In S. kaned (Ed.), UpToDate. https://www.uptodate.com/contents/overview-of-the-evaluation-of stroke/print?search=diagnostico

Cavichioli, M. G., Yugar-Toledo, J. C. \& Vilela-Martin J. F. (2014). Hypertensive emergency and ischemic and hemorrhagic stroke: current concepts of treatment. Rev Bras Hipertens. 21(4) 177- 183 https://docs.bvsalud.org/biblioref/2018/03/881314/rbh-v21n4_177-183.pdf

Figueiredo, M. M., Bichuetti, D. B. \& Gois, A. F. T. (2012) Evidências sobre diagnóstico e tratamento do acidente vascular encefálico no serviço de urgência. Diagn Tratamento. 17(12) 167-172. http://files.bvs.br/upload/S/1413-9979/2012/v17n4/a3328.pdf

Filho, J O \& Lansberg, M G. (2020) Neuroimaging of acute ischemic stroke. 2020. In S. Kasned (Ed.), UpToDate. https://www.uptodate.com/contents/neuroimaging-of-acute-ischemic-stroke/print?search=diagnostico

Fonseca L., Rosa M., Silva A., Maciel R., Volschan A. \& Mesquita E. (2013). Análise das barreiras à utilização de trombolíticos em casos de acidente vascular cerebral isquêmico em um hospital privado do Rio de Janeiro, Brasil. Cadernos de Saúde Pública. 29(12) 2487-2496 https://doi.org/10.1590/0102$311 \mathrm{X} 00131412$

Lima M. R., Paglioli R. \& Hoefel Filho J. R. (2012). Diagnóstico por imagem do acidente vascular encefálico / Image diagnosis of stroke. Acta méd. (Porto Alegre); 33(9). https://pesquisa.bvsalud.org/portal/resource/pt/biblio-881595

Kulesh A. A., Drobakha V. E. \& Shestakov, V. V. (2019). Doença cerebral dos pequenos vasos: classificação, manifestações clínicas, diagnóstico e características do tratamento. Neurologia, Neuropsiquiatria, Psicossomática. 11(3), 4-17. Https://doi.org/10.14412/2074-2711-2019-3S-4-17

Magalhães S. S. F. \& Oliveira, M. F. O. (2017). Comparação dos métodos de imagem (tomografia computadorizada e ressonância magnética) para o diagnóstico de acidente vascular encefálico. Bahiana Journals. 6(1) 81-89. https://www5.bahiana.edu.br/index.php/enfermagem/article/view/1258

Martins, M. (2010). Uso de medidas de comorbidades para predição de risco de óbito em pacientes brasileiros hospitalizados. Rev Saúde Pública. 44(3), 448456. https://doi.org/10.1590/S0034-89102010005000003

Mourao, A. M., Vicente, L. C. C., Chaves, T. S. \& Sant'anna, R. V. (2017). Profile of patients with a diagnosis of stroke attended at a hospital in minas gerais accredited in the care line. Rev Bras Neurol. 53(4),12-16. https://revistas.ufrj.br/index.php/rbn/article/view/14634

Pereira, A. S., Shitsuka, D. M., Parreira, F. J. \& Shitsuka, R. (2018) Metodologia da pesquisa científica [e-book]. Santa Maria, RS: UFSM, NTE, 2018. 1 ebook

Ribeiro, R., R, C., Bertolin, D., Cesarino, C., Kusumota, L., \& Fantini, J. (2016). Caracterização dos pacientes com acidente vascular encefálico atendidos na emergência. Arquivos De CiêNcias Da SaúDe, 23(4), 78-82. doi:10.17696/2318-3691.23.4.2016.463

Rolim, C.L.R.C. \& Martins, M. (2017) O uso de tomografia computadorizada nas internações por Acidente Vascular Cerebral. Rev Bras Epidemiol. 15(1) 179187. https://www.scielo.br/j/rbepid/a/KFNpCf4NCd8mhesT4FqJsHP/?lang=pt\&format=pdf

Soeiro, S. M. (2018). Papel protetor dos estrogênios na aterosclerose. Dissertação de mestrado, Faculdade de Farmácia da Universidade de Coimbra. http://hdl.handle.net/10316/84409

Yamashita, L., Fukujima, M.., Granitoff, N. \& Prado, G. (2004). Paciente com acidente vascular cerebral isquêmico já é atendido com mais rapidez no Hospital São Paulo. Arquivos de 62(1), https://www.researchgate.net/publication/26342240_Paciente_com_acidente_vascular_cerebral_isquemico_ja_e_atendido_com_mais_rapidez_no_Hospital_S ao_Paulo

Zerna, C., Thomalla, G. \& Campbell, B. C. V. (2018). Current practice and future directions in the diagnosis and acute treatment of ischaemic stroke. The Lanceta. 392(10154) 1247-1256. doi: 10.1016 / S0140-6736 (18) 31874-9 